\title{
Laboratory Studies of Lead Removal from Liquid Scintillator in Preparation for KamLAND's Low Background Phase
}

\author{
Gregory Keefer \\ Lawrence Livermore National Laboratory, Livermore, California 94550, USA
}

\begin{abstract}
The removal of Radon induced Lead from liquid scintillator was extensively studied in preparation for KamLAND's low background phase. This work presents the results from laboratory experiments performed at the University of Alabama and their implications for KamLAND and future low background experiments using carbon based liquid scintillator. It was observed that distillation was the most effective purification procedure and that one must consider a non-polar and non-ionic component of Lead in order to reach the levels of radio-purity required for these new class of ultra-low background experiments.
\end{abstract}

Keywords: KamLAND, Liquid Scintillator, Neutrino Oscillations, Solar Neutrinos, Purification, Distillation, Silica Gel PACS: 78.40.-q, 78.40.Kc, 81.20.Ym, 81.20.Rg, 81.05.Lg, 26.65.+t, 14.60.Pq

\section{INTRODUCTION}

Removal of intrinsic radio-isotopes from liquid scintillator (LS) was extensively studied in preparation for a lowbackground phase of KamLAND [1, 2]. The primary focus of these studies was to determine how to remove ${ }^{210} \mathrm{~Pb}$ from the KamLAND LS. If a large enough ${ }^{210} \mathrm{~Pb}$ reduction factor could be achieved, then this would be one of the primary components needed to provide KamLAND with the sensitivity to measure ${ }^{7} \mathrm{Be}$ solar $v_{\mathrm{e}}$. However, ${ }^{210} \mathrm{~Pb}$ was not the only intrinsic radioactive background that was inhibiting this measurement. Table 1 shows the status of the intrinsic radioactivity which was inhibiting a ${ }^{7} \mathrm{Be}$ solar $v_{\mathrm{e}}$ measurement. A lower ${ }^{210} \mathrm{~Pb}$ background would also reduce the correlated reactor [3] and Geo-Neutrino backgrounds [4]. This is done by reducing the primary correlated background, ${ }^{12} \mathrm{C}(\alpha, \mathrm{n}){ }^{16} \mathrm{O}$. Thus, a detailed program aimed at understanding the innate nature of ${ }^{210} \mathrm{~Pb}$ removal from organic LS was an important undertaking for the KamLAND experiment.

All future low energy solar neutrino experiments using carbon based LS are subject to very stringent intrinsic radioactive contaminant requirements. Background studies from KamLAND data indicated concentration of ${ }^{210} \mathrm{~Pb}$ needed to be reduced by a factor of $10^{5}$, down to an unprecedented level of $10^{-25} \mathrm{~g} / \mathrm{g}$, as indicated in Table 1 This level of radio-purity equates to less than 1 atom per $\mathrm{kg}$ of LS! It thus becomes increasingly difficult to perform measurements in the laboratory on such ultra-pure LS.

This paper reports results obtained from laboratory experiments designed to target the purification of ${ }^{212} \mathrm{~Pb}$ and ${ }^{214} \mathrm{~Pb}$ from a LS mixture containing $80.2 \%$ n-Dodecane, $19.8 \%$ 1,2,4-Trimethylbenzene (PC) and $1.36 \pm 0.03 \mathrm{~g} / \mathrm{l}$ of 2,5-Diphenlyoxazole (PPO) n-Dodecane, PC. The primary methods studied for Lead removal were adsorption, distillation, heating and chemical extraction. Many other methods were also tried but did not give encouraging results and include: water extraction, $\mathrm{pH}$ dependence of water extraction, isotope exchange and filtering.

\section{EXPERIMENTAL PROCEDURE}

The experimental studies detailed in this paper were designed to measure concentrations of ${ }^{212} \mathrm{~Pb}$ in the laboratory down to $10^{-22} \mathrm{~g} / \mathrm{g}$. Measurements at this concentration are not achievable with ${ }^{210} \mathrm{~Pb}$ in the laboratory. However, it was deemed necessary to forgo using ${ }^{210} \mathrm{~Pb}$ in exchange for understanding the underlying properties of the nucleus in LS at this low level of concentration. At a concentration of $10^{-20} \mathrm{~g} / \mathrm{g}$ the specific ${ }^{212} \mathrm{~Pb}$ activity is $0.83 \mathrm{~Bq} / \mathrm{l}$, a factor $1.8 \times 10^{4}$ larger than that of ${ }^{210} \mathrm{~Pb}$ at equal concentration.

The primary assumption used to compare purification techniques performed with ${ }^{212} \mathrm{~Pb}$ and ${ }^{214} \mathrm{~Pb}$ as apposed to using ${ }^{210} \mathrm{~Pb}$ was that all isotopes of Lead are borne in the same manner, via Radon decay. It was assumed that this 
TABLE 1. Measured radioactivity concentrations in KamLAND LS. The limit for ${ }^{39} \mathrm{Ar}$ was derived from solubility arguments [1].

\begin{tabular}{ll}
\hline Isotope & Concentrations [g/g] \\
\hline${ }^{14} \mathrm{C}$ & $(3.98 \pm 0.94) \times 10^{-18}$ \\
${ }^{39} \mathrm{Ar}$ & $<4.3 \times 10^{-21}$ \\
${ }^{40} \mathrm{~K}$ & $(1.30 \pm 0.11) \times 10^{-16}$ \\
${ }^{85} \mathrm{Kr}$ & $(6.10 \pm 0.14) \times 10^{-20}$ \\
${ }^{210} \mathrm{~Pb}$ & $(2.06 \pm 0.04) \times 10^{-20}$ \\
${ }^{232} \mathrm{Th}$ & $(8.24 \pm 0.49) \times 10^{-17}$ \\
${ }^{238} \mathrm{U}$ & $(1.87 \pm 0.10) \times 10^{-18}$ \\
\hline
\end{tabular}

method of introducing Lead into the LS was the key component in how the ${ }^{210} \mathrm{~Pb}$ was actually dispersed throughout the KamLAND LS.

As such, two commercially available Radon sources were purchased from Pylon Electronics. These Radon sources dispersed Radon into a glass bubbler fitted with a bubbling stone and filled with LS. The Radon was dispersed into the LS via a filtered Nitrogen carrier gas. The Radon decayed while inside the LS and the ${ }^{212} \mathrm{~Pb}$ was counted on a Germanium $(\mathrm{Ge})$ detector. There were several gamma lines and nuclei that were used to determine the initial concentration of ${ }^{212} \mathrm{~Pb}$ after bubbling. The ${ }^{212} \mathrm{~Pb}$ loaded $\mathrm{LS}$ was then transferred to the appropriate purification system and the empty Nalgene bottle, used for counting the initial LS, was again counted on the Ge detector to determine the residual ${ }^{212} \mathrm{~Pb}$ which adhered to the bottle walls ${ }^{1}$. After the ${ }^{212} \mathrm{~Pb}$ loaded LS was purified, it was counted on the Ge detector to determine the final activity. If needed, the LS was filtered prior to counting to remove any particulates from the purification process. In all instances, the procedural systematics were measured and taken into account. The purification factor is defined throught this work as the ratio of initial to final activity in the LS.

While this procedure works great for ${ }^{212} \mathrm{~Pb}$ loaded LS it is not exactly the same for ${ }^{214} \mathrm{~Pb}$ loaded LS as ${ }^{222} \mathrm{Rn}$ is the nuclei with the largest half-life. In order to understand the effects of ${ }^{214} \mathrm{~Pb}$ purification we needed to take into account the growth and decay properly. However, the procedure outlined above did not change, only the analysis of the data. For purification procedures which achieved large reduction factors gamma counting was not sufficient. Thus, the fast beta-gamma coincidence which exists in both the ${ }^{222} \mathrm{Rn}$ and ${ }^{220} \mathrm{Rn}$ chains allowed us to achieve two orders of magnitude greater sensitivity in our studies, down to $10 \mathrm{mBq} / \mathrm{l}$. Using these two methods of counting, and the different isotopes of Lead, we were able to observe purification of ${ }^{212} \mathrm{~Pb}$ and ${ }^{214} \mathrm{~Pb}$ in LS at concentrations of $10^{-17}-10^{-22}$ $\mathrm{g} / \mathrm{g}$ !

\section{EXPERIMENTAL RESULTS}

The first experiments performed involved water. This was done because a water extraction system already existed in the Kamioka mine (where the KamLAND experiment exists) and thus it would mean a minimal investment if we could develop a system involving water extraction to achieve our desired goals. Unfortunately water was not ideal. A reduction factor of $1.02-1.1$ was achieved with de-ionized (DI) water. The primary method was a re-circulation scheme in which the water was continuously circulated through the LS. The DI water was broken up into small bubbles before it entered the LS to provide a greater surface to volume ratio. To further the efforts of water, experiments were also performed which looked at the dependence on the ${ }^{212} \mathrm{~Pb}$ removal relative to the $\mathrm{pH}$. There was an observed dependence in that the the removal efficiency was higher with lower $\mathrm{pH}$. However, the overall increase with a lower $\mathrm{pH}$ was only $15 \%$ relative to DI water and thus was deemed ineffective.

Several other types of experiments were performed that did not yield great results. LS was passed over a bed of granular Lead shot and Lead granules under the assumption that given an infinite number of stable ${ }^{206} \mathrm{~Pb}$ atoms, there would occur an isotope exchange of the ${ }^{212} \mathrm{~Pb}$ in the LS. However, there was no effect observed beyond that expected

\footnotetext{
${ }^{1}$ This was actually a significant amount of activity. There was no correlation with initial activity observed and the absolute amount could be as much as half the initial activity. Thus, it was imperative to count the empty bottle to account for all the initial activity.
} 
TABLE 2. Measured ${ }^{212} \mathrm{~Pb}$ reduction factors in LS. Only statistical errors are quoted. Experimental investigation of the systematic error for 32-64 $\mu \mathrm{m}$ gel yielded $7.2 \%$. The error on the mass measurements was $0.05 \mathrm{~g}$.

\begin{tabular}{lll}
\hline Adsorbent Type & ${ }^{212}$ Pb Reduction Factor & Adsorbent Mass [g] \\
\hline Selecto, Lot \#301286301, Si-gel 32-63 $\mu \mathrm{m}$ & $19.4 \pm 0.47$ & 1.5 \\
Selecto, Lot \#306279301, Si-gel 100-200 $\mathrm{mm}$ & $15.38 \pm 0.24$ & 1.5 \\
Selecto, Lot \#102085402, Alusil 70 & $27.03 \pm 0.73$ & 1.5 \\
Selecto, Lot \#109110402, Alusil Plus & $8.33 \pm 0.07$ & 1.5 \\
Selecto, Lot \#108110403, Alusil NanoSmart & $8.20 \pm 0.07$ & 1.5 \\
Selecto, Lot \#900110401, Si-gel NanoSmart ACT & $3.58 \pm 0.01$ & 1.5 \\
Selecto, Lot \#900110401, Alusil Coarse & $28.57 \pm 0.82$ & 1.5 \\
Selecto, Lot \#107223405, Alusil 40 without K & $10.31 \pm 0.21$ & 2.0 \\
Aldrich, 3-(Diethylenetriamino) Propyl-Functionalized gel & $8.33 \pm 0.69$ & 10.0 \\
Aldrich, Triamine Tetraacetate-Functionalized gel & $11.11 \pm 0.12$ & 10.2 \\
Aerosil 200 & $8.26 \pm 0.07$ & 15.0 \\
SÜD-CHEMIE, Cu/Mn Catalyst T-2550 & $3.45 \pm 0.02$ & 0.5 \\
SÜD-CHEMIE, Cu/Mn Catalyst T-2550, Crushed & $26.32 \pm 0.69$ & 7.2 \\
Ca $\left(\text { PO }_{4}\right)_{2}$ & $6.54 \pm 6.54$ & 2.0 \\
\hline
\end{tabular}

for the purification system itself.

Filtering was a primary means of removing particulates from liquids and was used in the course of our studies. It was observed that filtering did purify the ${ }^{212} \mathrm{~Pb}$ from the LS but only a factor 1.1 reduction (10\%) was observed on a single pass. The largest reduction factors were observed in filtering when the carrier gas used to load the LS with ${ }^{212} \mathrm{~Pb}$ was not pre-filtered. This resulted in factors of 1.5 reduction and can be attributed to particulates in the gas stream "catching" the ionized ${ }^{212} \mathrm{~Pb}$ atom which is then easily removed via filtration. Once a pre-filter was placed on the Nitrogen carrier gas, the reduction factor from filtering was consistent. While multiple filtrations increased the reduction factor modestly it was not to the orders of magnitude needed for KamLAND.

Adsorption was the most well studied purification technique. This is because it was very reproducible, it yielded reasonably good purification results and it allowed us to look at more than just Lead reduction. Adsorption was performed with Silica Gel provided by Selecto Scientific. There were also experiment performed with other adsorption materials such as $\mathrm{Cu} / \mathrm{Mn}$ and Alusil. The results from these experiments are listed in Table 2. The details can be found in reference [1]. The primary observation from the silica gel experiments was that there was consistently approximately $5 \%$ of the ${ }^{212} \mathrm{~Pb}$ which could not be removed via silica gel extraction. The amount of silica, the type, the procedure, or any other physical observable could improve the purification beyond a factor 30 . Using silica gel it was also possible to obtain reduction factors for ${ }^{222} \mathrm{Rn},{ }^{218} \mathrm{Po},{ }^{212} \mathrm{Bi},{ }^{214} \mathrm{Bi}$ and ${ }^{212} \mathrm{Bi}$. These details can also be found in reference [1].

The adsorption experiments presented a problem in that, adsorption addresses an ionic or polar form of a molecule or atom. If the Lead was in the LS in a non-charged state, then adsorption and many other experiments addressing this species, such as water extraction, would never achieve reduction factors of $10^{5}$. Thus it was hypothesized that the ${ }^{212} \mathrm{~Pb}$ as in the LS in an organic form. To test this theory, chemical analysis was used to specifically attack an organic Lead molecule. It was found that using $\mathrm{FeCl}_{3}, \mathrm{SnCl}_{3}, \mathrm{MoS}_{2}$ and Thiol Resin all gave increased results relative to silica gel. $\mathrm{SiO}_{2}+\mathrm{FeCl}_{3}+\mathrm{SiO}_{2}$ was then performed in series and a factor 1250 reduction in ${ }^{212} \mathrm{~Pb}$ was achieved. The first $\mathrm{SiO}_{2}$ extraction was performed to remove the "known" amount of ionic/polar ${ }^{212} \mathrm{~Pb}$ from the LS. Then the chemical extraction was performed to address the non-polar form of ${ }^{212} \mathrm{~Pb}$. The final $\mathrm{SiO}_{2}$ extraction was to clean up in a sense, to remove any possibly broken organic bonds that were now polar. While all of these chemical treatments worked, they were destructive to the LS. The optics of the LS were completely destroyed. However, it did hint to a new component of Lead not addressed in typical purification techniques.

Another method used to test the organo-metallic Lead hypothesis was heating. Tetraethyllead actually decomposes at $200{ }^{\circ} \mathrm{C}$ and thus heating LS composed of Tetraethyllead would cause the lead to decompose into a polar form which could then be extracted using silica gel. Experiments performed using heating in series with silica gel extraction showed that the purification factor was boosted by a factor of 10 . The total ${ }^{212} \mathrm{~Pb}$ reduction found in heating plus silica gel extraction was $278 \pm 23$. Details can be found in reference [1]. 
The final purification technique addressed was distillation. Distillation actually takes care of several of the issues discussed above without knowing it a priori. The heating of the LS in the distillation flask will decompose any volatile organo-metallic components of Lead. The distillation process itself works under the principle of separation of states. It was found necessary to remove the first amount of distilled liquid, the low boiling point liquid, and discard it to achieve the best performance. Ideally this would contain water and all the low boiling point organo-metallic Lead components. It was further found not to distill all the distillate from the flask. The packing material used in the distillation column acts as an adsorption material and removes the charged Lead component. Thus, distillation was found to be the most effective. A single pass distillation was found to remove a factor $4 \times 10^{3}$. One has to be careful not to rapidly boil-over the LS as this will carry impure LS. Typical distillation speeds in the lab were on the order of $10 \mathrm{ml} / \mathrm{min}$. Multiple distillations succeeded in achieving measured reduction factor of $10^{4}$. The major downside to distillation is that it is very hard to reproduce. Measurements were performed on different apparatuses and even on the same apparatus and purification factors could vary by a factor of 10 .

\section{CONCLUSION}

These laboratory studies contributed to a major paradigm shift in how we viewed the ${ }^{210} \mathrm{~Pb}$ was dissolved in the LS. Experimental evidence indicated the Lead impurities were present in the LS in an ionic and an organo-metallic form. Experiments designed to address separately the ionic form and the organic form of ${ }^{210} \mathrm{~Pb}$ indicated that approximately 3-5\% of Lead was present in an organo-metallic state in the LS. Thus, in order to achieve the substantial radio-purity needed in the next generation experiments using organic based materials, these radioactive organo-metallic isotopes must be addressed.

The methods found most effective for purifying LS and its components were distillation and adsorption with a combination of heating. Distillation yielded the largest independent reduction factor for all isotopes studied, without destroying the optical properties of the LS. Thus, distillation was the method used to remove ${ }^{210} \mathrm{~Pb}$ from KamLAND LS and resulted in "The First Observation of ${ }^{7}$ Be Solar Neutrinos with KamLAND" [1].

\section{ACKNOWLEDGMENTS}

The majority of this work was performed under the direction of my advisor, Andreas Piepke, at The University of Alabama. The Tohoku Research Center for Neutrino Science also participated in performing independent analysis of many of these experiments.

\section{REFERENCES}

1. G. Keefer, First Observation of ${ }^{7}$ Be Solar Neutrinos with KamLAND, Ph.D. thesis, The University of Alabama, Tuscaloosa, Alabama (2009).

2. Y. Kishimoto, J. Phys. Conf. Ser. 120, 052010 (2008).

3. S. Abe, et al., Phys. Rev. Lett. 100, 221803 (2008), hep-ex/0801. 4589.

4. T. Araki, et al., Nature 436, 499 (2005). 